\title{
Evaluation of Rice (Oryza sativa L.) Genotypes for Drought Tolerance at Germination and Early Seedling Stage
}

\author{
M. M. Islam ${ }^{1}$, E. Kayesh ${ }^{2}$, E. Zaman ${ }^{1}$, T. A. Urmi ${ }^{3}$ and M. M. Haque ${ }^{1}$ \\ Departments of Agronomy ${ }^{1}$, Horticulture ${ }^{2}$, Soil Science ${ }^{3}$, Bangabandhu Sheikh Mujibur Rahman \\ Agricultural University, Gazipur-1706, Bangladesh \\ *Corresponding author and Email: moshiul@bsmrau.edu.bd
}

Received: 22 April 2018

Accepted: 23 June 2018

\begin{abstract}
Drought stress is a major constraint to the production and yield stability of crops. Rice (Oryza sativa L.) is considered as a drought-sensitive crop species. Within this species, there are considerable varietal differences in sensitivity to this environmental stress. An experiment was conducted at the laboratory of the Department of Agronomy, Bangabandhu Sheikh Mujibur Rahman Agricultural University (BSMRAU), Bangladesh during April to September 2016 to evaluate 100 rice genotypes for drought tolerance during germination and early seedling growth stage. The genotypes were tested against five levels of drought stress imposed by Polyethylene glycol 6000 (PEG-6000) @ 0, 5, 10, 15 and $20 \%$. The experiment was laid out in a complete randomized design with four replications. The results showed that with increasing water stress, germination in all the genotypes decreased from $95.8 \%$ in control to $6.6 \%$ in highest stress (20\% PEG) level. Seedling height and dry weight also decreased in all rice genotypes with the increase in water stress level. Based on Standard Evaluation System (SES), 18 genotypes were selected primarily. Among the 18 genotypes, Ratoil and Chinisakkar showed higher germination index, relative seedling height and relative seedling dry weight than the check drought tolerant BRRI dhan43 at 20\% PEG. Beside these, performance of Kumridhan, Pusur and Somondori was also well at this level compared to BRRI dhan43. The genotypes Ratoil, Chinisakkar, Kumridhan, Pusur and Somondori showed the best performance under drought condition.
\end{abstract}

Keywords: Drought stress, seed germination, seedling growth, rice genotypes, tolerance.

\section{Introduction}

Water is already a scarce commodity in many parts of the world, and predicted climate changes will aggravate the situation in future. Water deficit causes extensive loss to agricultural production worldwide, thus being a severe threat to sustainable agriculture. Rice plays a major role as a staple food, supporting more than three billion people and comprising $50 \%$ to $80 \%$ of their daily calorie intake (Khush, 2005). Drought stress severely impairs rice production. Drought affects more than 23 million hectares of rainfed rice in Asia (Kumbhar et al., 2015). The present and anticipated global food demands necessitate a significant increase in crop productivity on these less favourable rainfed lands. With diminishing water supplies for agriculture worldwide, the needs to improve drought adaptation of rice and to screen resistant varieties are becoming increasingly important (Pandey and Sukla, 2015). Understanding plant diversity 
is relevant to assess plant behaviour in relation to adaptation to drought-prone environments, and designing an effective phenotyping strategy requires thorough understanding of plant survival under stress (Sarkar et al., 2013). Therefore, assessment of genotypic variability under different water stress conditions is an important precondition for a successful screening program focused on drought tolerance.

Rice (Oryza sativa L.) is sensitive to water stress and shows several morphological changes at different growth stages in response to drought stress (Henry et al., 2016). These involve plant height reduction, leaf rolling, leaf senescence, stomatal closure, decreased leaf elongation and lower dry matter production (Kumar et al., 2015). However, the sensitivity of rice to drought or water stress varies with timing, duration, severity of drought stress, variety and the growth stage of rice (Sokoto and Muhammad, 2014). Drought stress induces the reduction of rice growth and development. Due to the reduction in turgor pressure under stress, cell growth is severely impaired (Taiz and Zeiger, 2006). Drought affects both elongation as well as expansion of growth, and inhibits cell enlargement more than cell division (Jaleel et al., 2009). It has been reported that seed germination and early seedling growth are potentially the most critical stages for water stress (Ahmad et $a l ., 2009)$ and many researchers also reported that drought stress impairs the seed germination (Swain et al., 2014) seedling height (Sokoto and Muhammad, 2014) and tiller number of rice (Bunnag and Pongthai, 2013). Farooq et al. (2010) stated that the common adverse effect of drought is the reduction in biomass production. It was also showed that drought significantly decrease fresh and dry weight of seedling (Ji et al., 2012).

However, the impact of drought stress on various morpho-physiological changes significantly differs among rice cultivars (Kumar et al., 2015) which underscore the importance of screening rice germplasm for drought tolerance. On the other hand, responses to environmental stress in plants are complex and multigenic, and the functions of many induced genes are still a matter of conjuncture. Because of this complexity, selection and breeding of droughttolerant genotypes are extremely difficult (Tirado and Cotter, 2010). Therefore, for easy selection of rice genotypes under drought stress, it is necessary to do a proper drought screening, which clearly distinguishes drought-susceptible genotypes from drought-tolerant genotypes (Swamy et al., 2012). Seed germination and seedling development is very important for early establishment of plants under stress condition. Selecting genotypes for rapid and uniform germination under water stress conditions can contribute towards early seedling establishment. Hence analysis of germination and seedling growth traits and their response to drought can be useful for selection of rice genotypes tolerant to drought. Drought scoring was also used as a primary criterion for screening rice genotypes for drought tolerance (IRRI, 2014). Leaf rolling with tip drying is one of the acclimation responses of rice and is used as a criterion for scoring drought tolerance. Leaf rolling with tip drying is hydronasty that leads to reduced light interception, transpiration and leaf dehydration (Kadioglu and Terzi, 2007). It may help in maintaining internal plant water status (Ha, 2014). If cell turgor is maintained under drought stress, it will result in delayed leaf rolling and drying. Modern rice cultivars tolerant to drought are few in number. However, it is essential to develop drought tolerant rice varieties to fulfill the future food demand for added population of the country. Therefore, the objective of the present study was to analyze genotypic variations in drought tolerance of rice at germination and early seedling stage.

\section{Materials and Methods}

A laboratory experiment was conducted at the Department of Agronomy, Bangabandhu Sheikh Mujibur Rahman Agricultural University (BSMRAU), Gazipur, Bangladesh during April to September, 2016. One hundred rice (Oryza sativa L.) genotypes along with check variety 
BRRI dhan43 were used in this experiment. Seeds of different genotypes were collected form drought-prone area of Bangladesh, Bangladesh Rice Research Institute (BRRI), Bangladesh Institute of Nuclear Agriculture (BINA), and also from Genetic Resources Unit of BSMRAU.

Seeds of each genotype were surface sterilized with $70 \%$ ethanol solution for 5 minutes. The seeds were then washed three times with sterilized distilled water. Germination assays were performed by evenly distributing the seeds in a 10-cm-diameter sterilized Petri dish with two layers of whatman No. 1 filter paper. Each dish was moistened with $10 \mathrm{ml}$ distilled water or uniform amounts of desired osmotic solutions to mimic drought stress. During screening, waterdeficit stress is artificially induced by desired strengths of polyethylene glycol 6000 (PEG6000; Sigma Chemicals). Polyethylene glycol has beenused to simulate water stress effects in plants (Swapna and Shylaraj, 2017).The experiment was laid out in a complete randomized design (CRD) with five levels of drought stress and four replications. Distilled water was used as a control (0 MPa) and osmotic potentials $-0.3,-0.6,-0.9$ and $-1.2 \mathrm{MPa}$ were created by adding PEG-6000 @ 5, 10, 15 and 20 $\mathrm{g}$ per $100 \mathrm{ml}$ distilled water. Four replicates of 50 seeds of each osmotic potential were used to assess the germination percentage. This experiment was carried out in growth chamber at $25 \pm 0.5^{\circ} \mathrm{C}$ and $80 \% \pm 1$ of relative humidity. The number of germinated seed was recorded at 24 hours interval. The seedling height and seedling dry weights were measured on the $14^{\text {th }}$ day. Seeds were considered germinated when both plumule and radicle extended to more than $2 \mathrm{~mm}$ from the seeds. The Standard Evaluation System (SES) for rice (IRRI, 1980)was used for screening of drought tolerant rice genotypes (Table 1).Visual scores for stress symptoms on scale 0 to 9 , where lower score denotes the tolerance and higher score denotes susceptible.

\subsection{Germination Index}

The germination index was calculated after final germinationusing the following equation:

$\mathrm{GI}=\frac{\text { Germination percentage in each treatment }}{\text { Germination percentage in the control }} \times 100$

\subsection{Seedling height and dry weight}

Ten seedling were chosen randomly and seedling height was measured. The lengths of seedling were measured with a ruler. Dry weight was determined after drying the seedlings at $70^{\circ} \mathrm{C}$ for 48 h. Data on germination and seedling characteristics for each treatment were compared with control for determining the drought tolerant rice genotypes.

Table 1.Standard Evaluation System (SES) for rice (IRRI, 1980)

\begin{tabular}{|c|c|}
\hline Description & Drought score \\
\hline No symptoms of stress effects & 0 \\
\hline Slight leaf rolling and tip drying & 1 \\
\hline $\begin{array}{l}\text { Leaf rolling and tip drying extended to } 1 / 4 \text { length in } 25 \% \text { of all leaves } \\
\text { (normally the older leaves) }\end{array}$ & 2 \\
\hline $\begin{array}{l}\text { Leaf rolling and tip drying extended to } 1 / 4 \text { length or more in at most } \\
50 \% \text { of all leaves }\end{array}$ & 3 \\
\hline $\begin{array}{l}\text { Leaf rolling and tip drying extended to } 1 / 4 \text { length or more in } 50 \% \text { of } \\
\text { all leaves with } 25 \% \text { of leaves fully rolled and dried }\end{array}$ & 4 \\
\hline $50 \%$ of all leaves fully rolled and dried & 5 \\
\hline $\begin{array}{l}\text { More than } 50 \% \text { but less than } 70 \% \text { of all leaves fully rolled and } \\
\text { dried }\end{array}$ & 6 \\
\hline Seventy percent of all leaves fully rolled and dried & 7 \\
\hline More than $70 \%$ of all leaves fully rolled and dried & 8 \\
\hline All plants apparently dead & 9 \\
\hline
\end{tabular}




\subsection{Relative seedling height}

The relative seedling height (RSH) was calculated using the following equation:

$\mathrm{RSH}(\%)=$

Plant height under drought condition

Plant height under control condition

\subsection{Relative dry weight}

The relative dry weight (RDW) was calculated using the following equation

RDW $(\%)=$

Total dry weight under drought condition $\times 100$

Total dry weight under control condition

\subsection{Statistical analysis}

The collected data were analyzed to assess their statistical significance. Statistix 10 program were used to perform statistical analysis. Means were separated by least significant difference (LSD).

\section{Results and Discussion}

\subsection{Visual scoring of drought injury at seedling} stage

Drought score is treated as an alternative approach to determine plant drought tolerance (Fen et al., 2015). Visual scoring is a reliable measure of tolerance for the estimation of oxidative damage in plants, and reflects dehydration of the plant tissue (Cabuslay et al., 2002). When water stress develops, the plants naturally have evolved a defensive mechanism for abbreviating the energy load on the leaf (Chaturvedi et al., 2012) and experienced leaf rolling and drying to reduce net radiation load on the leaf.

Table 2. Visual score at seedling stage of different rice genotypes under varied PEG levels.

\begin{tabular}{|c|c|c|c|c|c|}
\hline \multirow{3}{*}{ Genotypes } & \multicolumn{5}{|c|}{ Visual score } \\
\hline & Control & 5 & 10 & 15 & 20 \\
\hline & \multicolumn{5}{|c|}{$\%$ PEG } \\
\hline Dharial & 0.33 & 2.33 & 4.33 & 5.33 & 8.00 \\
\hline Majoaishe & 0.33 & 2.67 & 5.33 & 6.33 & 8.33 \\
\hline Pusur & 0.00 & 0.33 & 1.33 & 3.33 & 6.33 \\
\hline Mongthong & 0.00 & 1.00 & 3.00 & 4.33 & 7.33 \\
\hline Kaisha pajra & 0.33 & 2.00 & 4.00 & 5.33 & 8.33 \\
\hline Lal aus & 0.00 & 2.67 & 5.33 & 7.33 & 8.33 \\
\hline Sadaaus & 0.33 & 2.33 & 4.00 & 6.33 & 8.33 \\
\hline Saita & 0.00 & 0.33 & 2.33 & 4.33 & 7.33 \\
\hline Kumridhan & 0.00 & 0.00 & 1.33 & 3.33 & 6.00 \\
\hline Ratoil & 0.00 & 0.00 & 0.67 & 1.67 & 4.67 \\
\hline Sili & 0.00 & 1.33 & 4.00 & 5.33 & 8.33 \\
\hline Pidi 1 & 0.00 & 2.67 & 5.33 & 7.33 & 8.33 \\
\hline Shamraj & 0.33 & 1.33 & 4.33 & 5.33 & 8.33 \\
\hline Chinisakkar & 0.00 & 0.00 & 0.67 & 2.33 & 5.33 \\
\hline Somondori & 0.00 & 0.00 & 1.33 & 3.33 & 5.67 \\
\hline Nadinggoga pro & 0.33 & 0.33 & 5.00 & 6.33 & 8.33 \\
\hline Glongdhan & 0.00 & 0.33 & 5.33 & 6.00 & 8.33 \\
\hline BRRI dhan43 (CK) & 0.00 & 0.00 & 0.67 & 2.33 & 5.33 \\
\hline CV $(\%)$ & 300 & 51.35 & 23.38 & 12.74 & 8.77 \\
\hline $\operatorname{LSD}_{(0.05)}$ & 0.55 & 0.93 & 1.25 & 1.01 & 1.06 \\
\hline $\mathrm{SE}( \pm 0.05)$ & 0.2722 & 0.4581 & 0.6186 & 0.4969 & 0.5212 \\
\hline
\end{tabular}


In rice, leaf rolling and tip drying factor under drought stress was studied as one of the best criteria in estimating levels of drought tolerance in a large scale screening (Pandey and Shukla, 2015). In this study, the Standard Evaluation System (SES) for rice (IRRI, 1980) was used for screening of 100 rice genotypes at early seedling stage. Visual score 1 indicate highly tolerant, 2-3 indicate tolerant, 4-5 indicate moderately tolerant and 6-9 indicate susceptible to highly susceptible. Out of 100 genotypes only 18 genotypes were found tolerant at 5\% PEG. However, all the selected genotypes were found moderately tolerant at 10 and $15 \%$ PEG and only 2 genotypes were found moderately tolerant at $20 \%$ PEG (Table 2). Therefore, only 18 genotypes were considered for further discussion.

\subsection{Germination and germination index (GI)}

Germination is one of the most critical periods in the life cycle of plants. The effect of increasing concentrations of PEG on seed germination was measured to determine the tolerance of rice genotypes to water deficit. Seed germination was reduced as the PEG concentration increased in the nutrient solution and different PEG concentration had significant effect on percent seed germination of different rice genotypes (Table 3). The maximum percentage of germination was found in control condition and the minimum was at $20 \%$ PEG (Table 3 ).

Table 3. Germination percentage of different rice genotypes under varied PEG levels

\begin{tabular}{lccccc}
\hline & \multicolumn{5}{c}{ Germination $(\%)$} \\
\cline { 2 - 5 } Genotypes & Control & 5 & 10 & 15 & 20 \\
\cline { 2 - 5 } & $94.0 \mathrm{ab}$ & $85.2 \mathrm{c}$ & $72.5 \mathrm{~cd}$ & $45.3 \mathrm{~g}$ & $17.5 \mathrm{gh}$ \\
\hline Dharial & $92.0 \mathrm{ab}$ & $82.7 \mathrm{c}$ & $68.1 \mathrm{e}$ & $39.3 \mathrm{j}$ & $14.5 \mathrm{j}$ \\
Majoaishe & $94.6 \mathrm{ab}$ & $91.2 \mathrm{a}$ & $81.8 \mathrm{ab}$ & $59.0 \mathrm{~d}$ & $33.2 \mathrm{c}$ \\
Pusur & $91.7 \mathrm{ab}$ & $86.3 \mathrm{bc}$ & $75.3 \mathrm{c}$ & $48.4 \mathrm{f}$ & $21.6 \mathrm{e}$ \\
Mongthong & $90.0 \mathrm{~b}$ & $82.3 \mathrm{c}$ & $71.0 \mathrm{de}$ & $44.7 \mathrm{gh}$ & $16.8 \mathrm{gh}$ \\
Kaisha pajra & $92.7 \mathrm{ab}$ & $83.4 \mathrm{c}$ & $70.3 \mathrm{de}$ & $35.2 \mathrm{k}$ & $14.8 \mathrm{ij}$ \\
Lal aus & $92.2 \mathrm{ab}$ & $86.1 \mathrm{c}$ & $73.2 \mathrm{~cd}$ & $40.5 \mathrm{ij}$ & $16.6 \mathrm{gi}$ \\
Sadaaus & $95.8 \mathrm{a}$ & $86.7 \mathrm{bc}$ & $79.8 \mathrm{~b}$ & $55.3 \mathrm{e}$ & $28.2 \mathrm{~d}$ \\
Saita & $94.8 \mathrm{ab}$ & $91.7 \mathrm{a}$ & $83.8 \mathrm{a}$ & $62.3 \mathrm{bc}$ & $34.6 \mathrm{bc}$ \\
Kumridhan & $95.6 \mathrm{a}$ & $93.6 \mathrm{a}$ & $85.4 \mathrm{a}$ & $65.5 \mathrm{a}$ & $37.7 \mathrm{a}$ \\
Ratoil & $92.3 \mathrm{ab}$ & $86.8 \mathrm{bc}$ & $72.3 \mathrm{~cd}$ & $42.9 \mathrm{~h}$ & $19.7 \mathrm{f}$ \\
Sili & $90.1 \mathrm{~b}$ & $83.0 \mathrm{c}$ & $69.7 \mathrm{de}$ & $33.6 \mathrm{k}$ & $14.8 \mathrm{ij}$ \\
Pidi 1 & $93.1 \mathrm{ab}$ & $85.0 \mathrm{c}$ & $71.7 \mathrm{cde}$ & $42.7 \mathrm{hi}$ & $18.4 \mathrm{fg}$ \\
Shamraj & $94.7 \mathrm{ab}$ & $91.2 \mathrm{a}$ & $84.7 \mathrm{a}$ & $63.9 \mathrm{ab}$ & $36.8 \mathrm{a}$ \\
Chinisakkar & $94.7 \mathrm{ab}$ & $91.3 \mathrm{a}$ & $82.3 \mathrm{ab}$ & $61.3 \mathrm{c}$ & $34.8 \mathrm{bc}$ \\
Somondori & $92.4 \mathrm{ab}$ & $85.3 \mathrm{c}$ & $75.3 \mathrm{c}$ & $33.4 \mathrm{k}$ & $16.3 \mathrm{hij}$ \\
Nadinggoga pro & $92.6 \mathrm{ab}$ & $84.5 \mathrm{c}$ & $74.9 \mathrm{c}$ & $34.1 \mathrm{k}$ & $16.2 \mathrm{hij}$ \\
Glongdhan & $93.8 \mathrm{ab}$ & $90.5 \mathrm{ab}$ & $83.5 \mathrm{a}$ & $63.4 \mathrm{abc}$ & $36.4 \mathrm{ab}$ \\
BRRI dhan43 (CK) & 3.45 & 2.99 & 2.87 & 2.83 & 4.61 \\
CV (\%) & 5.33 & 4.31 & 3.63 & 2.26 & 1.82 \\
LSD (0.05) & 2.6270 & 2.1273 & 1.7888 & 1.1187 & 0.8963 \\
SE ( \pm 05 ) & & & & & 50
\end{tabular}

Note: Figure having common letter(s) in a column do not differ significantly at $5 \%$ level of significance. 
In general, germination was severely affected by 20\% PEG and all the selected genotypes had germination less than $40 \%$. However, differential tolerance of rice genotypes was observed; for example, Ratoil, Chinisakkar, Somondori and Kumridhan showed 37.7, 36.8, 34.8 and $34.6 \%$ germination in the presence of $20 \%$ PEG, whereas the values for Majoaishe and Pidi 1 in the same treatment were 14.5 and $14.8 \%$, respectively. The germination index (GI) also decreased with the increase in drought stress. In drought stress the highest GI was recorded at control and lowest at 20\% PEG concentration. However, the GI of Ratoil, Chinisakkar, Somondori and Kumridhan was found similar to that of the check BRRI dhan43 (Table 4). The results further indicated that these materials showed superiority over the rest selected genotypes in relation to germination. In the present investigation drought stress greatly affects seed germination, but the response intensity and adverse effect of stress depend on the genotypes (Table 3). It has been reported that drought stress adversely affect the seed germination, and seedling growth (Sokoto and Muhammad, 2014; Swain et al., 2014). Under water stress, low water potential is a determining factor for inhibiting seed germination (Wang et al., 2002). PEG is an osmotic agent, which play an important role in the regulation of mineral elements, hormone, protein metabolism and effects of signal transduction (Verslues et al., 1998). The main function of PEG is to slow down the moisture rate of seeds (Jiao et al., 2009).

Table 4.Germination index of different rice genotypes under varied PEG levels

\begin{tabular}{|c|c|c|c|c|c|}
\hline \multirow{3}{*}{ Genotypes } & \multicolumn{5}{|c|}{ Germination Index } \\
\hline & Control & 5 & 10 & 15 & 20 \\
\hline & \multicolumn{5}{|c|}{$\%$ PEG } \\
\hline Dharial & - & $90.6 \mathrm{~cd}$ & $77.1 \mathrm{efg}$ & $48.2 \mathrm{gh}$ & $18.6 \mathrm{gh}$ \\
\hline Majoaishe & - & $89.9 \mathrm{~d}$ & $74.0 \mathrm{~g}$ & $42.7 \mathrm{j}$ & $15.8 \mathrm{i}$ \\
\hline Pusur & - & $96.4 \mathrm{ab}$ & $86.5 \mathrm{a}$ & $62.4 \mathrm{~d}$ & $35.1 \mathrm{c}$ \\
\hline Mongthong & - & $94.1 \mathrm{a}-\mathrm{d}$ & $82.1 \mathrm{bc}$ & $52.8 \mathrm{f}$ & $23.6 \mathrm{e}$ \\
\hline Kaisha pajra & - & $91.4 \mathrm{a}-\mathrm{d}$ & $78.9 \mathrm{c}-\mathrm{f}$ & $49.7 \mathrm{~g}$ & $18.7 \mathrm{gh}$ \\
\hline Lal aus & - & $90.0 \mathrm{~d}$ & $75.8 \mathrm{fg}$ & $38.0 \mathrm{k}$ & $16.0 \mathrm{i}$ \\
\hline Sadaaus & - & $93.4 \mathrm{a}-\mathrm{d}$ & 79.4cde & $43.9 \mathrm{ij}$ & $18.0 \mathrm{ghi}$ \\
\hline Saita & - & $90.5 \mathrm{~d}$ & $83.3 \mathrm{~b}$ & $57.7 \mathrm{e}$ & $29.4 \mathrm{~d}$ \\
\hline Kumridhan & - & $96.7 \mathrm{ab}$ & $88.4 \mathrm{a}$ & $65.7 \mathrm{bc}$ & $36.5 \mathrm{bc}$ \\
\hline Ratoil & - & $97.9 \mathrm{a}$ & $89.3 \mathrm{a}$ & $68.5 \mathrm{a}$ & $39.4 \mathrm{a}$ \\
\hline Sili & - & $94.0 \mathrm{a}-\mathrm{d}$ & $78.3 \mathrm{def}$ & $46.5 \mathrm{hi}$ & 21.3ef \\
\hline Pidi 1 & - & $92.1 \mathrm{a}-\mathrm{d}$ & 77.4ef & $37.3 \mathrm{k}$ & $16.4 \mathrm{hi}$ \\
\hline Shamraj & - & $91.3 \mathrm{bcd}$ & 77.0efg & $45.9 \mathrm{hi}$ & $19.8 \mathrm{fg}$ \\
\hline Chinisakkar & - & $96.3 \mathrm{abc}$ & $89.4 \mathrm{a}$ & $67.5 \mathrm{abc}$ & $38.9 \mathrm{ab}$ \\
\hline Somondori & - & $96.4 \mathrm{ab}$ & $86.9 \mathrm{a}$ & $64.7 \mathrm{~cd}$ & $36.7 b c$ \\
\hline Nadinggoga pro & - & $92.3 \mathrm{a}-\mathrm{d}$ & $81.5 \mathrm{bcd}$ & $36.1 \mathrm{k}$ & $17.6 \mathrm{ghi}$ \\
\hline Glongdhan & - & $91.3 \mathrm{bcd}$ & $80.9 \mathrm{bcd}$ & $36.8 \mathrm{k}$ & $17.5 \mathrm{ghi}$ \\
\hline BRRI dhan43 (CK) & - & $96.5 \mathrm{ab}$ & $89.0 \mathrm{a}$ & $67.6 \mathrm{ab}$ & $38.8 \mathrm{ab}$ \\
\hline CV $(\%)$ & & 3.67 & 2.35 & 3.27 & 5.90 \\
\hline $\operatorname{LSD}_{(0.05)}$ & & 5.68 & 3.19 & 2.81 & 2.49 \\
\hline $\mathrm{SE}( \pm 0.05)$ & & 2.7987 & 1.5728 & 1.3836 & 1.2274 \\
\hline
\end{tabular}

Note: Figure having common letter(s) in a column do not differ significantly at 5\% level of significance. 
The inhibiting action of water stress on the rice germination was increased with PEG concentration increasing (Table 3). It seems that lowering the osmotic potential with PEG decrease water availability for seeds and then caused low germination. The physical process of water uptake leads to activation of metabolic process, as the dormancy of the seed is broken following hydration. Elevated drought stress slows dawn water uptake by seeds, thereby inhibiting their germination and root-shoot elongation.

\subsection{Seedling height and relative seedling height} Seedling height also decreased as the PEG concentration increased and seedling height of different rice varieties were significantly affected by water stress (Table 5). The maximum seedling height was observed in the controlled condition and the minimum in the highest drought stress level (Table 5). At all the drought levels, the maximum seedling height were found in Ratoil, Chinisakkar, Pusur, Kumridhan and Somondori (Table 5). Relative seedling height (RSH) was decreased with the increase in drought level. The highest RSH $(83.15 \%)$ was recorded at control and the lowest $(4.31 \%)$ was recorded at 20\% PEG concentration (Fig. 1). However, the \%RSH at 20\% PEG was far higher in Ratoil, Chinisakkar, Pusur, Kumridhan and Somondori other than check variety (Fig. 1). Under water stress, it has been shown that the inhibition of radicle emergence is mainly because of decrease in water potential gradient between the external environment and the seed and consequently impairs seedling height (Sokoto and Muhammad, 2014).

Table 5. Seedling height of different rice genotypes under varied PEG levels

\begin{tabular}{lccccc}
\hline \multirow{2}{*}{ Genotypes } & \multicolumn{5}{c}{ Seedling height $(\mathrm{cm})$} \\
\cline { 2 - 6 } & Control & 5 & 10 & 15 & 20 \\
\cline { 2 - 5 } Dharial & $21.46 \mathrm{abc}$ & $16.12 \mathrm{bc}$ & $9.94 \mathrm{de}$ & $5.01 \mathrm{f}$ & $1.38 \mathrm{def}$ \\
Majoaishe & $21.33 \mathrm{abc}$ & $14.56 \mathrm{def}$ & $8.27 \mathrm{hi}$ & $4.44 \mathrm{gh}$ & $0.92 \mathrm{hi}$ \\
Pusur & $20.22 \mathrm{~b}-\mathrm{f}$ & $15.71 \mathrm{bcd}$ & $10.46 \mathrm{~cd}$ & $6.51 \mathrm{c}$ & $2.04 \mathrm{bc}$ \\
Mongthong & $21.06 \mathrm{a}-\mathrm{d}$ & $14.31 \mathrm{efg}$ & $9.22 \mathrm{efg}$ & $5.51 \mathrm{e}$ & $1.48 \mathrm{~d}$ \\
Kaisha pajra & $19.94 \mathrm{c}-\mathrm{f}$ & $14.54 \mathrm{~d}-\mathrm{g}$ & $8.86 \mathrm{f}-\mathrm{i}$ & $4.79 \mathrm{fg}$ & $1.25 \mathrm{efg}$ \\
Lal aus & $19.27 \mathrm{ef}$ & $13.28 \mathrm{fgh}$ & $8.31 \mathrm{ghi}$ & $4.31 \mathrm{~h}$ & $0.88 \mathrm{i}$ \\
Sadaaus & $20.77 \mathrm{~b}-\mathrm{e}$ & $14.95 \mathrm{cde}$ & $9.11 \mathrm{e}-\mathrm{h}$ & $4.68 \mathrm{fgh}$ & $1.19 \mathrm{fg}$ \\
Saita & $21.81 \mathrm{ab}$ & $16.23 \mathrm{bc}$ & $10.56 \mathrm{bcd}$ & $6.06 \mathrm{~d}$ & $1.95 \mathrm{c}$ \\
Kumridhan & $22.44 \mathrm{a}$ & $17.85 \mathrm{a}$ & $11.59 \mathrm{a}$ & $7.22 \mathrm{a}$ & $2.25 \mathrm{ab}$ \\
Ratoil & $21.42 \mathrm{abc}$ & $17.81 \mathrm{a}$ & $11.46 \mathrm{ab}$ & $7.26 \mathrm{a}$ & $2.39 \mathrm{a}$ \\
Sili & $21.54 \mathrm{abc}$ & $14.31 \mathrm{efg}$ & $9.38 \mathrm{ef}$ & $4.98 \mathrm{f}$ & $1.45 \mathrm{de}$ \\
Pidi 1 & $19.23 \mathrm{ef}$ & $11.23 \mathrm{i}$ & $8.16 \mathrm{i}$ & $4.34 \mathrm{~h}$ & $0.91 \mathrm{hi}$ \\
Shamraj & $18.64 \mathrm{f}$ & $12.52 \mathrm{hi}$ & $8.89 \mathrm{f}-\mathrm{i}$ & $4.68 \mathrm{fgh}$ & $1.17 \mathrm{fg}$ \\
Chinisakkar & $21.33 \mathrm{abc}$ & $16.87 \mathrm{ab}$ & $11.38 \mathrm{abc}$ & $6.97 \mathrm{ab}$ & $2.22 \mathrm{ab}$ \\
Somondori & $20.84 \mathrm{a}-\mathrm{e}$ & $16.23 \mathrm{bc}$ & $10.65 \mathrm{a}-\mathrm{d}$ & $6.63 \mathrm{bc}$ & $2.08 \mathrm{bc}$ \\
Nadinggoga pro & $21.03 \mathrm{a}-\mathrm{d}$ & $13.26 \mathrm{gh}$ & $8.88 \mathrm{f}-\mathrm{i}$ & $4.67 \mathrm{fgh}$ & $1.13 \mathrm{gh}$ \\
Glongdhan & $19.52 \mathrm{def}$ & $12.42 \mathrm{hi}$ & $8.79 \mathrm{f}-\mathrm{i}$ & $4.69 \mathrm{fgh}$ & $1.21 \mathrm{fg}$ \\
BRRI dhan43(CK) & $20.61 \mathrm{~b}-\mathrm{e}$ & $16.47 \mathrm{~b}$ & $10.98 \mathrm{abc}$ & $6.89 \mathrm{abc}$ & $2.12 \mathrm{bc}$ \\
\hline CV (\%) & 4.81 & 5.23 & 5.86 & 4.48 & 8.66 \\
LSD (0.05) & 1.65 & 1.29 & 0.94 & 0.41 & 0.22 \\
SE ( \pm 0.05) & 0.8119 & 0.6369 & 0.4646 & 0.2023 & 0.1100 \\
\hline Note Figure & & & &
\end{tabular}

Note: Figure having common letter(s) in a column do not differ significantly at 5\% level of significance. 


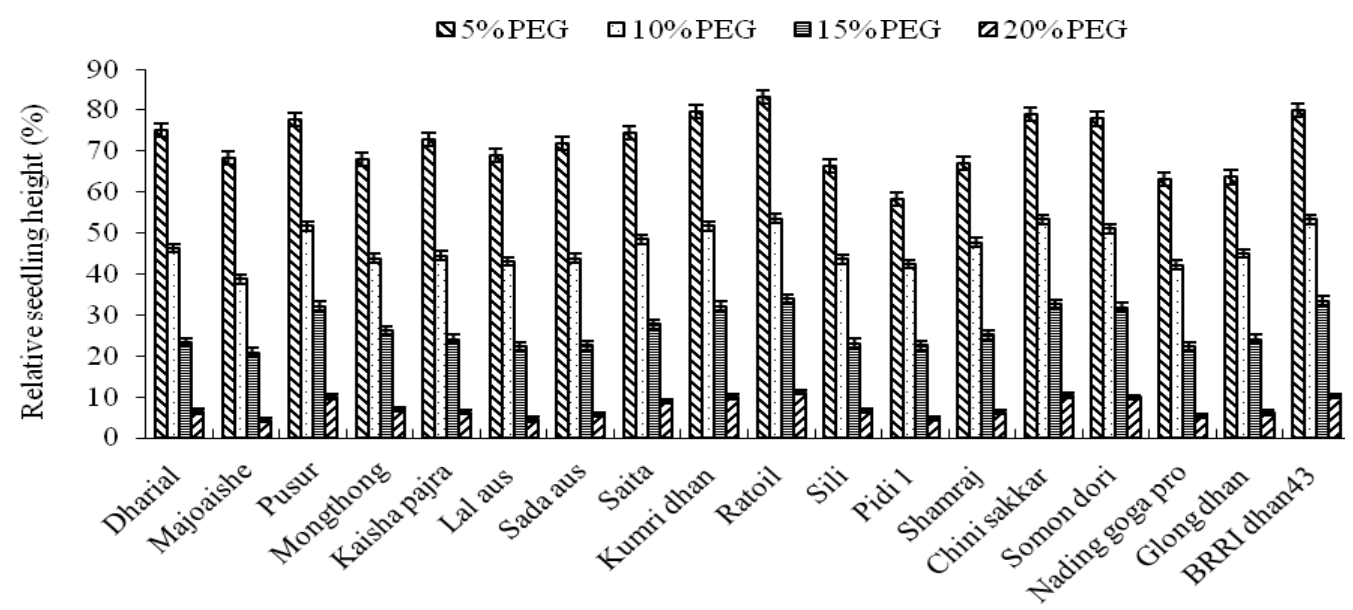

Genotypes

Figure 1. Relative seedling height (\%) of different rice genotypes under varied PEG level. Averages from three independent experiments are shown. Error bars represent the SE.

Table 6. Seedling dry weight of different rice genotypes under varied PEG levels

\begin{tabular}{lccccc}
\hline \multirow{2}{*}{ Genotypes } & \multicolumn{5}{c}{ Seedling dry weight (mg) } \\
\cline { 2 - 5 } & Control & 5 & 10 & 15 & 20 \\
\cline { 2 - 5 } Dharial & \multicolumn{5}{c}{$\%$ PEG } \\
Majoaishe & $10.10 \mathrm{~h}$ & $7.90 \mathrm{ij}$ & $4.30 \mathrm{~h}$ & $1.69 \mathrm{jk}$ & $0.38 \mathrm{~g}$ \\
Pusur & $8.50 \mathrm{i}$ & $6.10 \mathrm{k}$ & $3.60 \mathrm{i}$ & $1.36 \mathrm{k}$ & $0.14 \mathrm{~h}$ \\
Mongthong & $14.30 \mathrm{a}$ & $11.80 \mathrm{abc}$ & $7.60 \mathrm{~cd}$ & $3.54 \mathrm{~cd}$ & $1.25 \mathrm{~b}$ \\
Kaisha pajra & $13.60 \mathrm{abc}$ & $10.90 \mathrm{cde}$ & $6.05 \mathrm{ef}$ & $2.34 \mathrm{efg}$ & $0.61 \mathrm{~d}$ \\
Lal aus & $11.40 \mathrm{fg}$ & $8.80 \mathrm{hi}$ & $4.90 \mathrm{~g}$ & $1.98 \mathrm{hij}$ & $0.48 \mathrm{f}$ \\
Sadaaus & $10.80 \mathrm{gh}$ & $7.60 \mathrm{j}$ & $4.10 \mathrm{~h}$ & $1.66 \mathrm{jk}$ & $0.18 \mathrm{~h}$ \\
Saita & $12.90 \mathrm{bcd}$ & $10.10 \mathrm{efg}$ & $5.60 \mathrm{f}$ & $2.23 \mathrm{fgh}$ & $0.56 \mathrm{de}$ \\
Kumridhan & $11.70 \mathrm{efg}$ & $9.60 \mathrm{fgh}$ & $6.00 \mathrm{ef}$ & $2.69 \mathrm{e}$ & $0.85 \mathrm{c}$ \\
Ratoil & $14.60 \mathrm{a}$ & $12.40 \mathrm{ab}$ & $8.30 \mathrm{ab}$ & $3.98 \mathrm{ab}$ & $1.36 \mathrm{a}$ \\
Sili & $13.80 \mathrm{ab}$ & $12.80 \mathrm{a}$ & $8.70 \mathrm{a}$ & $4.13 \mathrm{a}$ & $1.37 \mathrm{a}$ \\
Pidi 1 & $12.60 \mathrm{cde}$ & $10.30 \mathrm{ef}$ & $5.70 \mathrm{f}$ & $2.13 \mathrm{ghi}$ & $0.50 \mathrm{ef}$ \\
Shamraj & $11.70 \mathrm{efg}$ & $9.10 \mathrm{gh}$ & $4.90 \mathrm{~g}$ & $1.81 \mathrm{ij}$ & $0.31 \mathrm{~g}$ \\
Chinisakkar & $10.80 \mathrm{gh}$ & $8.56 \mathrm{hij}$ & $4.80 \mathrm{~g}$ & $1.86 \mathrm{ij}$ & $0.35 \mathrm{~g}$ \\
Somondori & $12.90 \mathrm{bcd}$ & $12.10 \mathrm{ab}$ & $8.00 \mathrm{bc}$ & $3.83 \mathrm{abc}$ & $1.25 \mathrm{~b}$ \\
Nadinggoga pro & $13.80 \mathrm{ab}$ & $11.60 \mathrm{bc}$ & $7.40 \mathrm{~d}$ & $3.27 \mathrm{~d}$ & $1.19 \mathrm{~b}$ \\
Glongdhan & $13.60 \mathrm{abc}$ & $10.50 \mathrm{def}$ & $6.20 \mathrm{e}$ & $2.62 \mathrm{e}$ & $0.58 \mathrm{~d}$ \\
BRRI dhan43(CK) & $12.80 \mathrm{~b}-\mathrm{e}$ & $10.10 \mathrm{efg}$ & $5.90 \mathrm{ef}$ & $2.55 \mathrm{ef}$ & $0.57 \mathrm{~d}$ \\
\hline CV (\%) & $12.30 \mathrm{def}$ & $11.40 \mathrm{bcd}$ & $7.40 \mathrm{~d}$ & $3.68 \mathrm{bc}$ & $1.19 \mathrm{~b}$ \\
LSD (0.05) & 5.75 & 6.42 & 4.54 & 8.17 & 6.39 \\
SE ( \pm 0.05) & 1.18 & 1.07 & 0.46 & 0.36 & 0.08 \\
\hline Not Fig & 0.5794 & 0.5287 & 0.2253 & 0.1754 & 0.0380 \\
\hline
\end{tabular}

Note: Figure having common letter(s) in a column do not differ significantly at 5\% level of significance. 
3.4. Seedling dry weight and relative dry weight The seedling dry weight and relative dry weight (RDW) of different rice genotypes were influenced by drought stress. In all the genotypes the seedling dry weight and percent relative dry weight decreased due to increasing the PEG concentration (Table 6 and 7). At 20\% PEG, the highest RDW was found in Ratoil (9.93\%) and the lowest $(1.65 \%)$ was found in Majoaishe (Table 7). Chinisakkar (9.69\%), Kumridhan (9.32\%), Pusur $(8.74 \%)$, and Somondori $(8.62 \%)$ also perform better compared to others genotypes (Table 7). The result indicated that as the drought level increased, seedling dry weight and percent relative dry weight decreased. The RDW value of a plant reflects its vigour and is considered a good index of its exposure to stresses of all sorts (Xu et al., 2006). The RDW response of rice seedlings exposed to increasing PEG concentrations, revealed a decrease for seedling height (Table 5). This may reflect the impact of water stress on root cell development, which would likely impair nutrient uptake as well as having detrimental effects on photosynthesis, essential for biomass accumulation and therefore on shoot and root elongation. Water stress therefore appears to reduce the absorption and utilization of water to such an extent that the tolerance mechanisms employed by these plants in a drought are insufficient to maintain normal growth.

Table 7. Relative dry weight of different rice genotypes under varied PEG levels

\begin{tabular}{lccccc}
\hline \multirow{2}{*}{ Genotypes } & \multicolumn{5}{c}{ Relative dry weight (\%) } \\
\cline { 2 - 5 } & Control & 5 & 10 & 15 & 20 \\
\cline { 2 - 5 } Dharial & \multicolumn{5}{c}{ \%PEG } \\
Majoaishe & - & $78.22 \mathrm{e}$ & $42.57 \mathrm{~g}$ & $16.73 \mathrm{fg}$ & $3.76 \mathrm{~g}$ \\
Pusur & - & $71.76 \mathrm{f}$ & $42.35 \mathrm{~g}$ & $16.00 \mathrm{fgh}$ & $1.65 \mathrm{j}$ \\
Mongthong & - & $82.52 \mathrm{bc}$ & $53.15 \mathrm{~d}$ & $24.76 \mathrm{c}$ & $8.74 \mathrm{c}$ \\
Kaisha pajra & - & $77.19 \mathrm{cde}$ & $44.49 \mathrm{efg}$ & $17.21 \mathrm{f}$ & $4.49 \mathrm{e}$ \\
Lal aus & - & $42.98 \mathrm{fg}$ & $17.37 \mathrm{f}$ & $4.21 \mathrm{ef}$ \\
Sadaaus & - & $70.37 \mathrm{f}$ & $37.96 \mathrm{~h}$ & $15.37 \mathrm{~h}$ & $1.67 \mathrm{j}$ \\
Saita & - & $82.05 \mathrm{bcd}$ & $51.28 \mathrm{~d}$ & $22.99 \mathrm{~d}$ & $7.26 \mathrm{~d}$ \\
Kumridhan & - & $84.93 \mathrm{~b}$ & $56.85 \mathrm{c}$ & $27.26 \mathrm{~b}$ & $9.32 \mathrm{~b}$ \\
Ratoil & - & $92.75 \mathrm{a}$ & $63.04 \mathrm{a}$ & $29.93 \mathrm{a}$ & $9.93 \mathrm{a}$ \\
Sili & - & $81.75 \mathrm{bcd}$ & $45.24 \mathrm{ef}$ & $16.90 \mathrm{f}$ & $3.97 \mathrm{fg}$ \\
Pidi 1 & - & $77.78 \mathrm{e}$ & $41.88 \mathrm{~g}$ & $15.47 \mathrm{gh}$ & $2.65 \mathrm{i}$ \\
Shamraj & - & $79.26 \mathrm{cde}$ & $44.44 \mathrm{efg}$ & $17.22 \mathrm{f}$ & $3.24 \mathrm{~h}$ \\
Chinisakkar & - & $93.80 \mathrm{a}$ & $62.02 \mathrm{ab}$ & $29.69 \mathrm{a}$ & $9.69 \mathrm{ab}$ \\
Somondori & - & $84.06 \mathrm{~b}$ & $53.62 \mathrm{~d}$ & $23.70 \mathrm{~cd}$ & $8.62 \mathrm{c}$ \\
Nadinggoga pro & - & $77.21 \mathrm{e}$ & $45.59 \mathrm{ef}$ & $19.26 \mathrm{e}$ & $4.26 \mathrm{ef}$ \\
Glongdhan & - & $78.91 \mathrm{de}$ & $46.09 \mathrm{e}$ & $19.92 \mathrm{e}$ & $4.45 \mathrm{e}$ \\
BRRI dhan43 (CK) & - & $92.68 \mathrm{a}$ & $60.16 \mathrm{~b}$ & $29.92 \mathrm{a}$ & $9.67 \mathrm{ab}$ \\
\hline CV (\%) & & 2.44 & 3.28 & 3.86 & 4.38 \\
LSD $(0.05)$ & & 3.29 & 2.65 & 1.34 & 0.41 \\
SE ( \pm 0.05$)$ & 1.6216 & 1.3072 & 0.6603 & 0.2027 \\
\hline
\end{tabular}

Note: Figure having common letter(s) in a column do not differ significantly at $5 \%$ level of significance. 


\section{Conclusions}

In conclusion, rice genotypes were very sensitive to drought stress. From the results of present investigation it can also be concluded that with increasing levels of water stress, seed germination and early seedling growth were adversely affected in all rice genotypes. However, for all the physiological parameters like germination percentage, seedling height, dry weight of seedling varietal differences were recorded and the difference was maximum towards higher stress levels. In response to water stress Ratoil, Chinisakkar, Pusur and Kumridhan showed better performance in terms of germination, relative seedling height and relative dry weight as compared to others.

\section{Acknowledgement}

We are most grateful to Research Management Committee (RMC) of BSMRAU, Gazipur, Bangladesh for financial support to complete this research.

\section{References}

Ahmad S., Ahmad R., Ashraf MY., Ashraf M., Waraich EA. 2009.Sunflower (Helianthus Annuus L.) response to drought stress at germination and seedling growth stages. Pakistan Journal of Botany, 41:647-54.

Bunnag S.,Pongthai P. 2013. Selection of rice (Oryza sativa L.) cultivars tolerant to drought stress at the vegetative stage under field conditions. American Journal of Plant Science, 4(9): 1701-1708.

CabuslayGS., Ito O., Alejar AA. 2002. Physiological evaluation of responses of rice (Oryza sativa L.) to water deficit. Plant Science, 163(4): 815-827.

ChaturvediGS., Singh A., Bahadur R. 2012. Screening techniques for evaluating crop germplasm for drought tolerance. Plant Archive, 12(1): 11-18.

Farooq M., Kobayashi N., Ito O., Wahid A.,
Serraj R. 2010. Broader leaves result in better performance of indica rice under drought stress. Journal of Plant Physiology, 167(13): 1066-1075.

Fen LL., Ismail MR., Zulkarami B., Rahman MSA., Ismail MR. 2015. Physiological and molecular characterization of drought responses and screening of drought tolerant rice varieties. Bioscience Journal, 31(3): 709-718.

Ha PTT. 2014. Physiological responses of rice seedlings under drought stress. Journal of Science and Development, 12(5): 635640.

Henry A., Wehler R., Grondin A., Franke R., Quintana M. 2016. Environmental and physiological effects on grouping of drought tolerant and susceptible rice varieties related to rice (Oryza sativa) root hydraulics under drought. Annals of Botany, 118(4): 711-724.

International Rice Research Institute (IRRI). 1980. Standard Evaluation System for Rice, 2nd edition, International Rice Research Institute, Los Bafios, The Philippines, 44 pp.

International Rice Research Institute (IRRI). 2014. Standard Evaluation System for Rice. 5th edn. Los Banos, the Philippines: International Rice Research Institute.

Jaleel CA., Manivannan P., Wahid A., Farooq M., Al-Juburi, HJ, Somasundaram R., Panneerselvam R. 2009. Drought stress in plants: A review on morphological characteristics and pigments composition. International Journal of Agricultural Biology, 11: 100-105.

Ji KX., Wang YY., Sun WN., Lou QJ., Mei HW., Shen SH., Chen H. 2012. Droughtresponsive mechanisms in rice genotypes with contrasting drought tolerance during reproductive stage. Journal of Plant Physiology, 169(4): 336-344.

Jiao SY., Li YQ., Shayila SH., Chen XL. 2009. 
Seeds Germination and Seedling Growth about 3 Pennisetum Ornamental Grasses under Drought Stress. Acta Botany Boreal.-Occident Sin, 29(2): 0308-0313.

Kadioglu A., Terzi R. 2007. A dehydration avoidance mechanism: Leaf rolling. Botany Review, 73(4): 290-302.

Khush GS. 2005. What it will take to feed 5.0 billion rice consumers in 2030. Plant Molecular Biology, 59(1): 1-6.

Kumar R., Sreenu K., Singh N., Jain N., Singh NK., Rai V. 2015. Effect of drought stress on contrasting cultivars of rice. International Journal of Tropical Agriculture, 33(2): 1559-1564.

Kumbhar SD., Kulwal PL., Patil JV., Sarawate CD., Gaikwad AP., Jadhav AS. 2015. Genetic diversity and population structure in landraces and improved rice varieties from India. Rice Science, 22(3): 99-107.

Pandey V., Shukla A. 2015. Acclimation and tolerance strategies of rice under drought stress. Rice Science, 22(4): 147-161.

Sarkar RK., Mahata KR., Singh DP. 2013. Differential responses of antioxidant system and photosynthetic characteristics in four rice cultivars differing in sensitivity to sodium chloride stress. Acta Physiology of Plant, 35(10): 2915-2926.

SokotoMB., Muhammad A. 2014. Response of rice varieties to water stress in Sokoto, Sudan Savannah, Nigeria. Journal of Bioscience Med, 2: 68-74.

Swain P., Anumalla M., Prusty S., MarndiBC., Rao GJN. 2014. Characterization of some Indian native land race rice accessions for drought tolerance at seedling stage. Australian Journal of Crop Science, 8(3): 324-331.
Swamy BPM., Kumar A. 2012. Sustainable rice yield in water-short drought-prone environments: Conventional and molecular approaches. In: Lee $\mathrm{T}$ S. Irrigation Systems and Practices in Challenging Environments. German: InTech.

Swapna S., Shylaraj KS. 2017. Screening for Osmotic Stress Responses in Rice Varieties under Drought Condition. Rice Science, 24(5): 253-263.

Taiz L., Zeiger E. 2006. Plant Physiology.4th edn. Sunderland, MA: Sinauer Associates: 7-64.

Tirado R., Cotter J. 2010. Ecological farming: Drought-resistant agriculture. In: Erwood S, Truchi N, Stabinsky D. Greenpeace Research Laboratories Technical Note. Amsterdam, Netherland: the Netherlands Greenpeace International.

Verslues PE., Ober ES., Sharp RE. 1998. Root growth an d oxygen relation at low water potentials impact of oxygen availability in polyethylene glycol solution. Plant Physiology, 116: 1403-1412.

Wang JG., Chen GC., Zhang CL. 2002. The effect s of water stress on soluble protein content, the activity of SOD, POD and CAT of two eco types of reeds (Phragmitescommunis). Acta Botany Boreal Occident Sin, 22(3): 561-565.

Xu SG., Wang JH., Bao LJ. 2006. Effect of Water Stress on Seed Germination and Seedling Growth of Wheat. Journal of Anhui Agricultural Science, 34: 57845787. 